\title{
BILIARY DISEASE
}

\section{Gall bladder dysmotility: a risk factor for gall stone formation in hypertriglyceridaemia and reversal on triglyceride lowering therapy by bezafibrate and fish oil}

\author{
I J A M Jonkers, A H M Smelt, M Ledeboer, M E Hollum, I Biemond, F Kuipers, \\ F Stellaard, R Boverhof, A E Meinders, C H B W Lamers, A A M Masclee
}

See end of article for authors' affiliations

Correspondence to: Dr A H M Smelt,

Department of General

Internal Medicine, Leiden

University Medical Centre,

PO Box 9600, 2300 RC

Leiden, the Netherlands;

A.H.M.Smelt@lumc.nl

Accepted for publication 25 June 2002

\begin{abstract}
Background and aim: The aim of this study was to unravel the mechanisms responsible for the increased risk of gall stone disease in hypertriglyceridaemia (HTG) and to compare the effects of triglyceride lowering therapy by bezafibrate and fish oil on determinants of cholelithiasis (biliary lipid composition and gall bladder motility) in HTG patients.

Patients and methods: Gall bladder motility (ultrasonography) was studied postprandially and during infusion of cholecystokinin (CCK). Determinants of cholelithiasis and serum lipids were compared between nine HTG patients and 10 age, sex, and body mass index matched normolipidaemic controls. The effects of bezafibrate and fish oil in HTG patients were studied in a randomised cross over trial. Results: HTG patients showed 14-fold higher serum triglyceride (TG) levels than controls. Biliary lipid composition, fasting gall bladder volumes, and CCK levels did not differ between HTG patients and controls. Gall bladder emptying was reduced in HTG patients compared with controls during CCK infusion $(-22 \%)$ as well as in response to a meal $(-37 \%$; both $p<0.001)$. Postprandial CCK levels were significantly higher in HTG patients. Both bezafibrate and fish oil reduced serum TG levels $1-68 \%$ and $-51 \%$ v baseline, respectively; both $\mathrm{p}<0.01)$. Fasting CCK levels were not affected whereas CCK induced gall bladder emptying increased during bezafibrate $(+29 \% ; p<0.001)$ and tended to increase on fish oil therapy $(+13 \% ; p=0.07)$. Postprandial gall bladder motility improved on bezafibrate and fish oil $(+47$ and $+25 \% v$ baseline, respectively; both $p<0.02)$ at least partly due to increased gall bladder sensitivity to CCK (both $p<0.05 v$ baseline). Bezafibrate but not fish oil increased the molar ratio of cholesterol to bile acids $(+40 \% ; p \leqslant 0.05)$ but no effects on cholesterol saturation index were seen with either treatment.

Conclusions: We suggest that impaired gall bladder motility occurs in HTG patients due to decreased sensitivity to CCK, which may add to the enhanced risk of gall stone disease in HTG patients. Triglyceride lowering therapy by both fish oil and bezafibrate improve gall bladder dysmotility without adversely affecting biliary cholesterol saturation.
\end{abstract}

E ndogenous hypertriglyceridaemia (HTG) is a multifactorial disorder of very low density lipoprotein (VLDL) metabolism, resulting in elevated serum triglyceride (TG) concentrations and low levels of high density lipoprotein (HDL) cholesterol. HTG is associated with cardiovascular disease, ${ }^{1}$ pancreatitis, ${ }^{2}$ and cholesterol gall stone formation. ${ }^{34}$

Both changes in biliary lipid composition (supersaturation with cholesterol) and gall bladder dysmotility are critical factors involved in the pathogenesis of cholesterol gall stones. ${ }^{56}$ In patients with HTG, bile appears to be supersaturated with cholesterol compared with controls. ${ }^{78}$ However, in these studies, ${ }^{78}$ HTG patients were compared with controls that were not matched for body mass index (BMI). As biliary cholesterol saturation is increased in obesity ${ }^{9}$ and HTG is frequently accompanied by obesity, ${ }_{10}^{10}$ the observed supersaturated bile in HTG may be related to the presence of obesity rather than to HTG itself. To date, it is not known whether gall bladder motility contributes to the increased risk of cholelithiasis in HTG. Therefore, the first aim of our study was to compare biliary lipid composition and gall bladder motility between HTG patients and age, sex, and BMI matched normolipidaemic controls.

Fibrates are the first choice TG lowering drugs for HTG patients to prevent cardiovascular disease. ${ }^{11}$ However, despite their profound TG lowering capacity, fibrates have been shown to increase the risk of cholelithiasis ${ }^{12-14}$ by increasing biliary cholesterol saturation. ${ }^{15}$ As the risk of cholelithiasis is already increased in $\mathrm{HTG},{ }^{3}$ treatment with fibrates could enhance the risk of cholelithiasis and subsequently pancreatitis even further. Fish oil, known for its profound hypotriglyceridaemic capacity, ${ }^{16}$ decreases biliary cholesterol saturation in patients with gall stone disease. ${ }^{17}$ Thus fish oil may be a therapeutic alternative to fibrates in HTG patients in exerting similar lipid lowering properties without adversely influencing the risk of gall stone formation. Therefore, a second aim of this study was to compare the effects of bezafibrate and fish oil on serum lipids and both biliary lipid composition and gall bladder motility as risk factors for the development of gall stone disease in patients with HTG.

\section{METHODS}

Patients and control subjects

The study population consisted of nine unrelated male patients with endogenous HTG who were recruited from the lipid outpatient clinic of the Leiden University Medical Centre and 10 normolipidaemic, age, sex, and BMI matched healthy control subjects who were recruited in response to a

Abbreviations: HTG, hypertriglyceridaemia; CCK, cholecystokinin; TG, triglyceride; (V)LDL, (very) low density lipoprotein; HDL, high density lipoprotein; CSI, cholesterol saturation index; BMI, body mass index. 
newspaper advertisement. The diagnosis of endogenous HTG was based on the mean of two fasting blood samples obtained after following, for at least eight weeks, a step 1 diet from the National Cholesterol Education Program. ${ }^{18}$ Diagnostic criteria for endogenous HTG were: total serum TG $>4.0 \mathrm{mmol} / \mathrm{l}$, VLDL cholesterol $>1.0 \mathrm{mmol} / \mathrm{l}$, and low density lipoprotein (LDL) cholesterol $<4.5 \mathrm{mmol} / \mathrm{l}$. Exclusion criteria were: homozygosity for apolipoprotein E2, secondary hyperlipidaemia (renal, liver, or thyroid disease, fasting glucose $>7.0 \mathrm{mmol} / \mathrm{l}$, and alcohol consumption $>40 \mathrm{~g} /$ day), and a medical history of cardiovascular disease, pancreatitis, or gall stones. The presence of asymptomatic gall stones or sludge was excluded by ultrasound. If patients used lipid lowering therapy before the study onset, this was stopped for at least six weeks prior to the study. None of the participants took any medication known to affect biliary cholesterol saturation or gall bladder motility.

\section{Study design}

The study started with assessment of baseline values in both controls and hypertriglyceridaemic patients: at visit 1 , duodenal bile was sampled and gall bladder motility was assessed during infusion of cholecystokinin (CCK) whereas at visit 2 (one week later) fasting blood samples were taken for assessment of serum lipids, and postprandial gall bladder motility was determined. Thereafter, HTG patients were randomised to receive in a crossover fashion either bezafibrate (Bezalip retard; Hoffmann-La Roche Ltd, Basel, Switzerland) $400 \mathrm{mg}$ once daily or fish oil 5 g/day (Triomar; Lube, Hadsund, Denmark; containing $3.6 \mathrm{~g} \omega-3$ fatty acids (eicosapentaenoic acid (C20:5) $1.9 \mathrm{~g}$ and docosahexaenoic acid (C22:6) $1.1 \mathrm{~g}$ )) for seven weeks. These two treatment periods were separated by a six week washout period without lipid lowering medication. At the onset and in week 7 of the treatment periods, fasting blood samples were obtained. Bile was sampled and CCK induced gall bladder motility was performed in week 6 whereas postprandial gall bladder motility was investigated in week 7 of each treatment period. Informed consent was obtained from each participant and the protocol was approved by the institutional medical ethics committee.

\section{Serum lipids}

Ultracentrifugation was performed to determine serum VLDL, LDL, and HDL cholesterol levels. ${ }^{19}$ Triglyceride and cholesterol concentrations were measured enzymatically using commercially available kits.

\section{CCK induced gall bladder motility}

Gall bladder volumes measured with real time ultrasonography (Toshiba, 3.75 MHz transducer) were calculated by the sum of cylinders method, as described previously. ${ }^{20}$ Gall bladder volumes were measured at predefined time intervals as an estimation of gall bladder motility. CCK induced gall bladder motility was studied during a continuous intravenous infusion of CCK (Ferring, Limhamn, Sweden) at a dose of 1.0 IDU/kg body weight/h for 45 minutes at 8.00 am after an overnight fast. Gall bladder volumes were assessed before $(t=0$ minutes $)$ and during intravenous infusion of CCK at $\mathrm{t}=15,30$, and 45 minutes. At $\mathrm{t}=0$ and $\mathrm{t}=45$ minutes, blood samples were taken for plasma CCK determination.

\section{Postprandial gall bladder motility}

Postprandial gall bladder motility was studied after ingestion of a standardised meal at $08.00 \mathrm{am}$ after an overnight fast. For determination of postprandial gall bladder motility, gall bladder volumes were measured before ( $\mathrm{t}=0$ minutes) and 10,20 , $30,45,60,75,90,105$, and 120 minutes after ingestion of a standardised meal (0-10 minutes; $780 \mathrm{kcal}, 50 \mathrm{~g}$ fat, $42 \mathrm{~g}$ protein, and $38 \mathrm{~g}$ carbohydrates). At the same intervals, blood samples were taken for plasma CCK determination.

\section{Bile sampling procedure}

Duodenal bile sampling was performed using a polyvinyl multilumen tube at 08.00 am after an overnight fast. The tube was introduced transnasally and placed in the horizontal part of the duodenum using a guide wire. Correct position of the tube was verified by fluoroscopy. After duodenal intubation, bile was sampled through this tube at 10 minute intervals after induction of gall bladder contraction by continuous intravenous infusion of CCK at a dose of $1.0 \mathrm{IDU} / \mathrm{kg}$ body weight/h for 45 minutes. This infusion resulted in strong gall bladder emptying $(>70 \%)$ in all participants. Bile fractions were immediately stored on ice.

\section{Biliary lipid analysis}

Only the darkest most concentrated bile fraction was used for analysis. Total lipids were extracted from bile by the Bligh and Dyer procedure. ${ }^{21}$ Cholesterol concentration was determined fluorimetrically using the cholesterol oxidase method. ${ }^{22}$ Phospholipids were measured spectrophotometrically after release of phosphate with $\mathrm{HclO}_{4}{ }^{23}$ Total bile salt concentration was determined in whole bile using the modified $3 \alpha$ hydroxysteroid dehydrogenase method. ${ }^{24}$ Bile acid composition in duodenal bile was determined by gas chromatography after enzymatic hydrolysis of bile acid conjugates and derivatisation of free acids. ${ }^{25}$

Cholesterol saturation index (CSI) was calculated assuming a total lipid concentration of $10 \mathrm{~g} / \mathrm{dl}$, using Carey's critical tables. ${ }^{26}$ The fatty acid species of biliary phospholipids were analysed by gas liquid chromatography. Total lipids were extracted from native bile ( $15 \mu \mathrm{l}$ ) to which $100 \mu \mathrm{g}$ heptadecanoic acid (C17:0) was added as internal standard with methanol/hexane $(4: 1 \mathrm{v} / \mathrm{v})$ and transmethylated with acetylchloride. ${ }^{27}$ The fatty acid methyl esters were extracted with hexane and analysed by gas liquid chromatography on a $50 \mathrm{~m} \times 0.2 \mathrm{~mm}$ OV-1 capillary column (HP-Ultra l; Agilent Technologies, Amstelveen, the Netherlands). ${ }^{28}$ Fatty acids were identified on the basis of retention times and cochromatography with authentic standards.

\section{Plasma cholecystokinin determination}

Plasma CCK was measured by a sensitive and specific radioimmunoassay. ${ }^{29}{ }^{30}$ The detection limit of the assay is 0.1 $\mathrm{pmol} / \mathrm{l}$ of plasma. The intra-assay variation ranged from $4.6 \%$ to $11.5 \%$ and the interassay variation ranged from $11.3 \%$ to $26.1 \%{ }^{29}$ Interference of lipid enriched plasma of HTG patients with binding of CCK to CCK antibodies was excluded.

\section{Data and statistical analysis}

Results are expressed as means (SEM). Gall bladder emptying was calculated as percentage of basal fasting gall bladder volume. Gall bladder emptying was evaluated in relation to the duration of the experiment as well as by quantification of the maximal measured gall bladder emptying. Integrated incremental values for plasma CCK secretion were calculated as the area under the plasma concentration curve after subtraction of the basal value at $\mathrm{t}=0$ minutes.

Differences in single parameters between HTG patients and controls were tested using the Mann-Whitney U test, whereas differences in HTG patients between baseline conditions and bezafibrate or fish oil therapy were evaluated using analysis of variance (ANOVA) with the post hoc LSD test.

Gall bladder emptying and postprandial CCK levels were analysed by multiple analysis of variance (MANOVA) for differences between HTG patients and controls. Differences in gall bladder emptying and postprandial CCK levels in HTG patients at baseline compared with after bezafibrate and fish oil therapy were analysed with multiple analysis of variance (MANOVA) with the post hoc LSD test.

In addition, gall bladder sensitivity to CCK was studied in response to CCK infusion as well as with regression analysis 
Table 1 Characteristics of controls and hypertriglyceridaemic patients at baseline and on bezafibrate and fish oil therapy

\begin{tabular}{|c|c|c|c|c|}
\hline \multirow[b]{3}{*}{ Age (y) } & \multirow[b]{2}{*}{ Controls } & \multicolumn{3}{|c|}{ Hypertriglyceridaemic patients } \\
\hline & & Baseline & Bezafibrate & Fish oil \\
\hline & $51.9 \quad(2.3)$ & $52.6 \quad(2.7)$ & & \\
\hline Body mass index $\left(\mathrm{kg} / \mathrm{m}^{2}\right)$ & $27.2(0.9)$ & $27.8 \quad(0.6)$ & $28.0 \quad(0.7)$ & $28.2(0.7)$ \\
\hline Total cholesterol (mmol/l) & $5.20(0.32)$ & $8.32(1.31)^{*}$ & $6.03(0.54) \dagger$ & $6.95(1.16)$ \\
\hline Total triglycerides (mmol/l) & $1.09(0.21)$ & $15.56(3.34)$ & $4.90(1.31) \dagger$ & $7.58(2.85) \dagger$ \\
\hline VLDL cholesterol (mmol/I) & $0.29(0.09)$ & $5.86(1.28)^{*}$ & $2.22(0.60) \dagger$ & $3.51(1.09) \dagger$ \\
\hline VLDL triglycerides $(\mathrm{mmol} / \mathrm{l})$ & $0.72(0.18)$ & $13.80(3.19)^{*}$ & $3.86(1.22) \dagger$ & $5.97(2.09) \dagger \ddagger$ \\
\hline LDL cholesterol (mmol/l) & $3.98(0.31)$ & $1.74(0.22)^{*}$ & $3.12(0.29) \dagger$ & $2.71(0.26) \dagger$ \\
\hline HDL cholesterol (mmol/l) & $1.12(0.07)$ & $0.63(0.05)^{*}$ & $0.74(0.05)$ & $0.66(0.05)$ \\
\hline
\end{tabular}

Values are mean (SEM).

${ }^{*} p \leqslant 0.05$ compared with controls; $\dagger p \leqslant 0.05$ compared with hypertriglyceridaemic patients at baseline; $¥ p \leqslant 0.05$ compared with hypertriglyceridaemic patients on bezafibrate.

(V)LDL, (very) low density lipoprotein; HDL, high density lipoprotein.

(random effect model with random intercept and slope), characterising the relationship between postprandial gall bladder emptying and integrated incremental plasma CCK levels in all participants at five time intervals: 0, 0-30 minutes, 0-60 minutes, 0-90 minutes, and 0-120 minutes. $p$ values of 0.05 were considered statistically significant.

\section{RESULTS}

\section{Baseline characteristics}

Age, sex, and BMI did not differ between HTG patients and controls. By definition, HTG patients had higher serum total TG and VLDL-TG concentrations than controls but other lipoprotein fractions also differed from those of controls (table 1).

\section{Effects of bezafibrate and fish oil on serum lipids in HTG patients}

Serum lipid levels in HTG patients did not differ between the first "baseline" determination and measurements before start of the bezafibrate and fish oil treatment periods. Therefore, only serum lipid levels at the end of these periods were compared (table 1). Both bezafibrate and fish oil decreased total TG, VLDL-TG, total cholesterol, and VLDL cholesterol whereas LDL and HDL cholesterol increased (table 1). Lipid lowering effects did not differ between bezafibrate and fish oil, except for VLDL-TG, which were lower on bezafibrate $(-35 \% v$ fish oil; $\mathrm{p}<0.05)$.

\section{CCK induced gall bladder emptying}

Fasting gall bladder volumes and CCK levels did not differ significantly between controls and HTG patients at baseline (fasting gall bladder volume 21.3 (1.8) and 18.5 (2.2) ml; and fasting CCK levels 0.5 (0.2) and 1.1 (0.1) pmol/l, respectively). Comparison between groups showed no difference in integrated incremental CCK levels in HTG patients at baseline compared with controls ( 409 (72) v 375 (73) pM $\times 45$ minutes). CCK induced gall bladder emptying, measured over 45 minutes however, was significantly lower in HTG patients than in controls $(-22 \% ; \mathrm{p}<0.001)$ and maximum CCK induced gall bladder emptying tended to be lower also (76 (6) $v 90(17) \% ; \mathrm{p}=0.07$ ) (fig 1).

Fasting gall bladder volumes and both fasting and integrated incremental CCK levels were not significantly different between HTG patients at baseline and during bezafibrate or fish oil treatment (fasting gall bladder volume 18.5 (2.2), 17.1 (1.6), and 19.1 (1.9) ml, respectively; fasting CCK levels $1.1(0.1), 1.1(0.2)$, and $0.8(0.2) \mathrm{pmol} / \mathrm{l}$, respectively; integrated incremental CCK 409 (72), 495 (76), and 506 (105) $\mathrm{pM} \times 45$ minutes, respectively). Compared with HTG patients at baseline, both bezafibrate and fish oil increased CCK induced gall bladder emptying, measured over 45 minutes $(+29 \%$, $\mathrm{p}<0.001$ and $+13 \%, \mathrm{p}=0.07)$. Maximum CCK induced gall

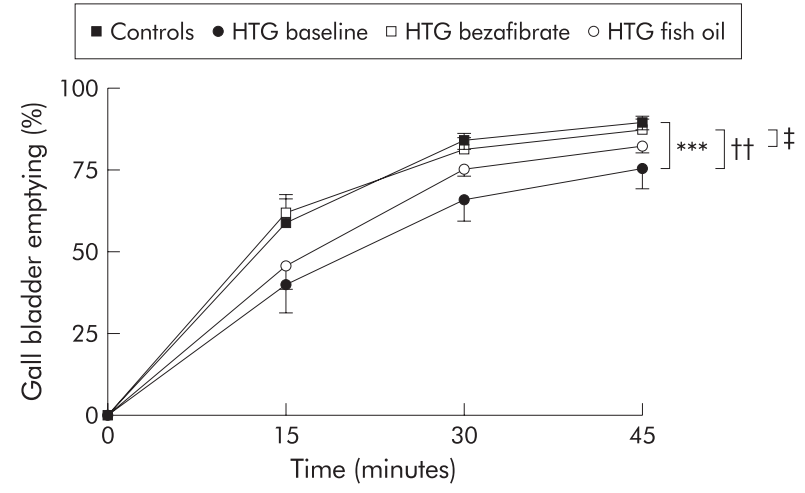

Figure 1 Cholecystokinin (CCK) induced gall bladder emptying motility in controls and hypertriglyceridaemic (HTG) patients at baseline, and on receiving bezafibrate and fish oil. Significant differences in CCK induced gall bladder emptying: ${ }^{* *} p=0.001$ between controls and HTG patients at baseline; $\dagger \dagger p<0.01$ between bezafibrate and baseline in HTG patients; $\ddagger p=0.02$ between bezafibrate and fish oil in HTG patients.

bladder emptying non-significantly increased on both bezafibrate and fish oil therapy $(+14 \%$ and $+8 \% v$ baseline; both $\mathrm{p}>0.09$ ) (fig 1). Compared with fish oil, bezafibrate resulted in higher gall bladder emptying over 45 minutes on exogenous CCK ( $+14 \%$; $p=0.02$ ) (fig 1 ) and a non-significant higher maximum gall bladder emptying (87 (3) v 82 (4)\%).

\section{Postprandial gall bladder motility}

Fasting gall bladder volumes and CCK levels did not differ between controls and HTG patients at baseline; neither did they differ from values obtained during assessment of CCK induced gall bladder emptying (table 2). Comparison between both groups showed higher postprandial CCK levels over 120 minutes (average CCK level 2.18 (0.09) and 1.29 (0.11) pmol/l for HTG patients and controls; $\mathrm{p}=0.045$ ) and a tendency to higher integrated incremental CCK levels in HTG patients at baseline compared with controls (272 (42) v 168 (32) $\mathrm{pM} \times 120$ minutes; $p=0.07)$. Concurrently, a lower postprandial gall bladder emptying over 120 minutes $(-37 \% ; \mathrm{p}<0.001)$ and a lower maximum gall bladder emptying $(-44 \% v$ controls; $\mathrm{p}<0.01$ ) were observed in HTG patients compared with controls (fig 3 ).

Fasting gall bladder volumes and both fasting and integrated incremental CCK levels were not different in HTG patients at baseline or during bezafibrate or fish oil treatment, and did not differ from values obtained during assessment of CCK induced gall bladder emptying (table 2). Postprandial CCK levels increased during bezafibrate therapy (average CCK level 2.57 (0.09) and 2.18 (0.09) pmol/l for HTG patients on 
Table 2 Cholecystokinin (CCK) induced gall bladder emptying and postprandial gall bladder emptying

\begin{tabular}{|c|c|c|c|c|}
\hline & \multirow[b]{2}{*}{ Controls } & \multicolumn{3}{|c|}{ Hypertriglyceridaemic patients } \\
\hline & & Baseline & Bezafibrate & Fish oil \\
\hline \multicolumn{5}{|l|}{ CCK induced gall bladder emptying } \\
\hline Fasting gall bladder volume (ml) & $21.3(1.8)$ & $18.5(2.2)$ & $17.1(1.6)$ & $19.1(1.9)$ \\
\hline Residual gall bladder volume (ml) & $2.2(0.3)$ & $4.2(1.0)$ & $2.1(0.5)$ & $3.4(1.0)$ \\
\hline Gall bladder emptying over $45 \mathrm{~min}(\%)$ & $58(3)^{* *}$ & $45 \quad(2)$ & $58(2)^{* *}$ & $51 \quad(2) \dagger$ \\
\hline Maximum gall bladder emptying (\%) & $90 \quad(17)$ & $76 \quad(6)$ & $87 \quad(3)$ & $82 \quad(4)$ \\
\hline Fasting CCK levels (pmol/li) & $0.5(0.2)$ & $1.1(0.1)$ & $1.1(0.2)$ & $0.8(0.2)$ \\
\hline Integrated incremental CCK levels (pM×45min) & 375 (73) & $409 \quad(72)$ & $495 \quad(76)$ & $506 \quad(105)$ \\
\hline \multicolumn{5}{|l|}{ Postprandial gall bladder emptying } \\
\hline Fasting gall bladder volume (ml) & $22.6(1.6)$ & $19.0(1.5)$ & $20.9(2.6)$ & $22.4(2.3)$ \\
\hline Residual gall bladder volume (ml) & $8.4(1.3)^{* *}$ & $11.9(1.1)$ & $8.5(1.5)^{* *}$ & $10.4(1.4)$ \\
\hline Gall bladder emptying over $120 \mathrm{~min}$ & $32(2)^{* *}$ & 20 (2) & $30(2)^{* \star}$ & $25(2)^{* \star} \dagger$ \\
\hline Maximum gall bladder emptying (\%) & $64(4)^{* *}$ & $35 \quad(6)$ & $61(14)^{* *}$ & 50 (7) \\
\hline Fasting CCK levels (pmol/l) & $0.2(0.1)$ & $0.9(0.4)$ & $0.7(0.2)$ & $0.9(0.3)$ \\
\hline Maximum CCK levels (pmol/l) & $2.9(0.3)$ & $3.4(0.4)$ & $3.8(0.3)$ & $3.7(0.3)$ \\
\hline Maximum CCK increase (pmol/l) & $2.7(0.3)$ & $2.5(0.3)$ & $3.2(0.3)$ & $2.9(0.3)$ \\
\hline Integrated incremental CCK levels (pM×120 min) & $168 \quad(32)$ & $272 \quad(42)$ & $329 \quad(45)$ & $302 \quad(42)$ \\
\hline
\end{tabular}

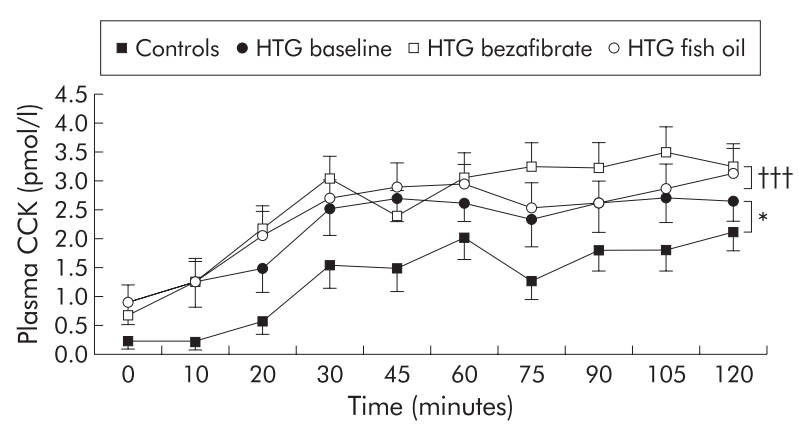

Figure 2 Postprandial cholecystokinin (CCK) levels in controls and hypertriglyceridaemic (HTG) patients at baseline, and after bezafibrate and fish oil therapy. Significant differences in postprandial CCK levels: * $p<0.05$ between controls and HTG patients at baseline; †††p=0.001 between bezafibrate and baseline in HTG patients.

bezafibrate $v$ baseline; $\mathrm{p}=0.001$ ) (fig 2) whereas maximum CCK levels, maximum CCK increase, and incremental integrated CCK levels did not differ between both periods (table 2). In contrast, fish oil did not affect postprandial CCK levels, maximum CCK levels, maximum CCK increase, or incremental integrated CCK levels. Compared with HTG patients at baseline, both bezafibrate and fish oil increased postprandial gall bladder emptying over 120 minutes $(+47 \%$ and $+25 \%$, respectively; both $\mathrm{p}<0.02$ ) and maximum gall bladder emptying $(+74 \%$ and $+43 \% \quad v$ HTG patients at baseline, respectively; both $\mathrm{p}<0.05)$. Compared with fish oil, bezafibrate increased postprandial gall bladder emptying $(+17 \% ; p=0.035)$ and non-significantly increased maximum gall bladder emptying (61 (14) v 50 (7)\% for bezafibrate and fish oil, respectively) (fig 3 ).

\section{Gall bladder sensitivity to CCK}

Regression analysis showed that postprandial gall bladder emptying was correlated with integrated incremental plasma CCK levels in controls and HTG patients at baseline, and during bezafibrate and fish oil treatment (all $p<0.001$ ). The slope of this line, representing gall bladder sensitivity to CCK, was significantly higher in controls than in HTG patients at baseline $(\beta=+0.10(0.04) \% \times \mathrm{pM} / \mathrm{min} v$ HTG patients at baseline; $\mathrm{p}<0.01$ ) (fig 2). Compared with baseline, both bezafibrate and fish oil improved gall bladder sensitivity to CCK $(\beta=+0.10$ (0.02) $\% \times \mathrm{pM} / \mathrm{min}$ and $\beta=+0.05(0.02) \% \times \mathrm{pM} / \mathrm{min} v$ baseline,

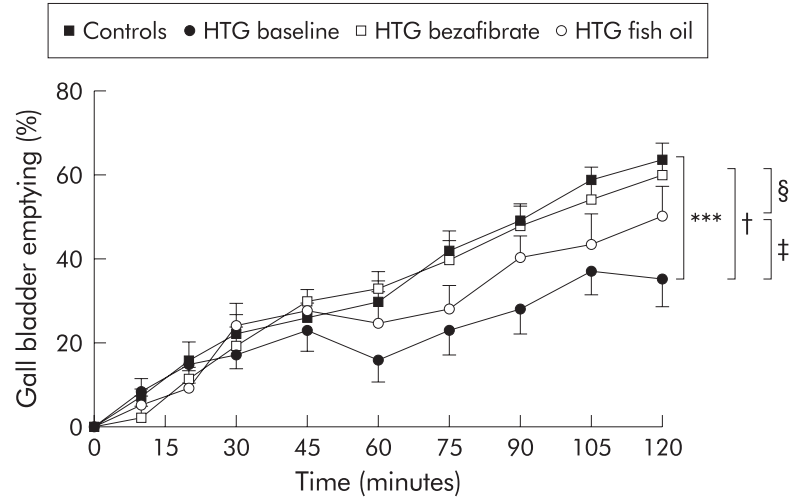

Figure 3 Postprandial gall bladder motility in controls and hypertriglyceridaemic (HTG) patients at baseline, and after bezafibrate and fish oil therapy. Significant differences in postprandial gall bladder emptying: ${ }^{* * *} p<0.001$ between controls and HTG patients at baseline; $\uparrow p<0.02$ between bezafibrate and baseline in HTG patients; $¥ p<0.02$ between fish oil and baseline in HTG patients; $\S p=0.035$ between bezafibrate and fish oil in HTG patients.

respectively; both $\mathrm{p}=0.05)$. In comparison with fish oil, bezafibrate improved gall bladder sensitivity to CCK ( $\beta=$ $+0.05(0.02) \% \times \mathrm{pM} / \mathrm{min} v$ fish oil; $\mathrm{p}=0.01$ ) (fig 4).

\section{Biliary lipid composition}

CSI exceeded 1 in both HTG patients and controls, indicating the presence of bile slightly supersaturated with cholesterol. However, no significant differences were observed in biliary lipid composition between HTG patients and controls (table $3)$.

Compared with baseline, bezafibrate increased the molar percentage cholesterol and decreased the molar percentage bile acids $(\mathrm{p}=0.05$ and $\mathrm{p}=0.06$, respectively), resulting in a higher molar ratio of cholesterol to bile acids $(+35 \% v$ baseline; $\mathrm{p}<0.05$ ). No effect was seen of bezafibrate on biliary bile acid composition (table 3 ). The molar percentage phospholipids and the molar ratio of phospholipids to bile acids tended to increase on bezafibrate $(p=0.16$ and $p=0.14)$. As a net result, CSI did not significantly change. In addition, bezafibrate slightly, but significantly, changed the proportions of $\alpha$-linoleic ( $\mathrm{Cl} 8: 3$, table 4 ) present in biliary phospholipids.

Intake of fish oil did not affect molar percentage cholesterol, bile acids, or phospholipids significantly, nor did it affect molar 


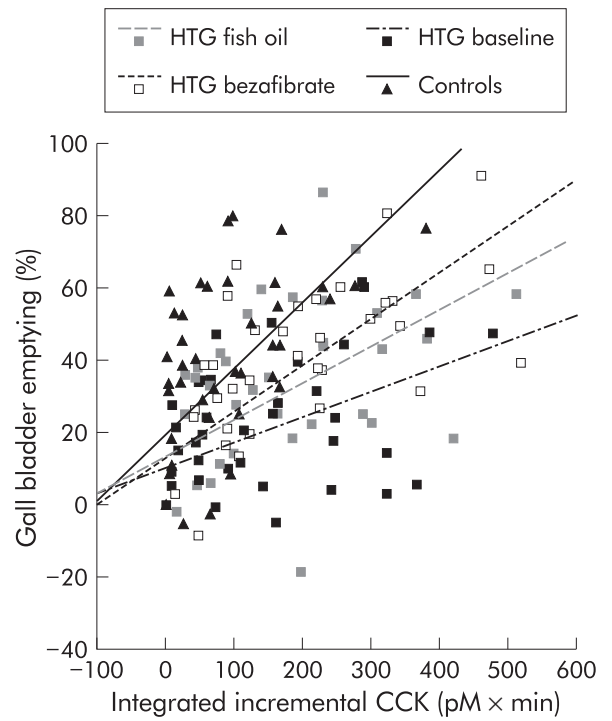

Figure 4 Gall bladder sensitivity to cholecystokinin (CCK) in controls and hypertriglyceridaemic (HTG) patients at baseline, and after bezafibrate and fish oil therapy. HTG patients had decreased gall bladder sensitivity to CCK compared with controls $(p<0.01)$. Both bezafibrate and fish oil significantly increased gall bladder sensitivity to CCK compared with HTG patients at baseline $(p<0.00$ and $p<0.05$, respectively). Gall bladder sensitivity to CCK was significantly higher in HTG patients on bezafibrate than on fish oil therapy $(p=0.01)$.

ratios between these three biliary components. Compared with baseline, fish oil altered the fatty acid distribution of biliary phospholipids: the $\omega-3$ phospholipid fatty acids fractions
(C20:5; C22:5; C22:6) increased at the expense of the linoleic acid (C18:2) fraction (table 3 ).

Comparison of biliary lipid composition between fish oil and bezafibrate showed an increase in molar percentage cholesterol and molar ratio of cholesterol to bile acids on bezafibrate therapy $(+32 \%$ and $+35 \% v$ fish oil, respectively; both $\mathrm{p}=0.05)$ (table 3$)$.

\section{DISCUSSION}

This study shows that biliary lipid composition does not differ between HTG patients and controls. In contrast with bezafibrate, fish oil does not increase biliary cholesterol content in HTG patients. In comparison with controls, HTG patients have impaired gall bladder motility during both exogenous CCK administration and in response to a meal, which reverses on TG lowering therapy by both bezafibrate and fish oil.

In HTG patients, bile was supersaturated with cholesterol, corroborating previous studies. ${ }^{78}$ However, in contrast with these reports, ${ }^{78}$ we did not observe a significant difference in CSI between HTG patients and normolipidaemic controls. This discrepancy might be due to a significantly lower BMI in these control groups compared with HTG patients ${ }^{78}$ whereas in our study HTG patients and controls had similar BMI values. A high BMI is associated with increased biliary cholesterol saturation. ${ }^{9}$ If HTG patients display supersaturated bile, this is most likely attributable to the co-occurrence of obesity rather than to the presence of HTG per se.

Fibrates increase the risk of cholelithiasis by increasing biliary cholesterol saturation ${ }^{15}$ due to enhanced biliary cholesterol secretion ${ }^{31}$ and possibly impaired bile acid synthesis. ${ }^{32} \mathrm{We}$ also observed a significant increase in the molar ratio of cholesterol to bile acids on bezafibrate therapy. However, this increased molar ratio did not coincide with a significant

Table 3 Biliary lipid composition

\begin{tabular}{|c|c|c|c|c|}
\hline & \multirow[b]{2}{*}{ Controls } & \multicolumn{3}{|c|}{ Hypertriglyceridaemic patients } \\
\hline & & Baseline & Bezafibrate & Fish oil \\
\hline Cholesterol (mol\%) & $4.20(0.27)$ & $4.92(0.43)$ & $6.28(1.96)^{*} \dagger$ & $4.74(0.59)$ \\
\hline Phospholipids (mol\%) & $10.73(1.22)$ & $11.69(1.60)$ & $15.57(1.70)$ & $14.93(2.28)$ \\
\hline Bile acids (mol\%) & $85.07(1.26)$ & $83.39(1.73)$ & $78.16(1.52)$ & $80.33(2.36)$ \\
\hline Molar ratio cholesterol to bile acids $\times 100$ & $4.96(0.33)$ & $5.96(0.60)$ & $8.04(0.84)^{*} \dagger$ & $5.96(0.36)$ \\
\hline Molar ratio cholesterol to phospholipids & $0.46(0.06)$ & $0.49(0.08)$ & $0.46(0.08)$ & $0.55(0.24)$ \\
\hline Molar ratio phospholipids to bile acids & $0.13(0.02)$ & $0.14(0.02)$ & $0.20(0.03)$ & $0.19(0.03)$ \\
\hline Cholesterol saturation index & $1.05(0.09)$ & $1.11(0.12)$ & $1.19(0.17)$ & $0.97(0.14)$ \\
\hline Cholic acid ( $\%$ of total bile acids) & $38.78(3.29)$ & $35.96(1.19)$ & $37.35(2.91)$ & $33.79(2.22)$ \\
\hline Chenodeoxycholic acid ( $\%$ of total bile acids) & $34.82(2.31)$ & $37.12(3.56)$ & $40.05(4.41)$ & $37.71(3.35)$ \\
\hline Deoxycholic acid (\% of total bile acids) & $26.40(5.42)$ & $26.92(3.31)$ & $22.60(5.01)$ & 28.50 (4.39) \\
\hline
\end{tabular}

Values are mean (SEM).

${ }^{*} p \leqslant 0.05$ compared with hypertriglyceridaemic patients at baseline; $\uparrow p \leqslant 0.05$ versus fish oil.

Table 4 Fractions of biliary phospholipid fatty acids

\begin{tabular}{|c|c|c|c|c|}
\hline & \multirow[b]{2}{*}{ Controls } & \multicolumn{3}{|c|}{ Hypertriglyceridaemic patients } \\
\hline & & Baseline & Bezafibrate & Fish oil \\
\hline Palmitic acid $(\mathrm{Cl6}$ :0) & $38.48(0.29)$ & $39.06(0.37)$ & $39.41(0.36)$ & $39.74(0.32)$ \\
\hline Palmitoleic acid (C16:1) & $2.27(0.34)$ & $1.70(0.15)$ & $2.29(0.44)$ & $1.45(0.19) \dagger$ \\
\hline Oleic acid (C18:1) & $11.27(0.63)$ & $9.77(0.47)$ & $10.82(0.44)$ & $8.96(0.45) \dagger$ \\
\hline Linoleic acid (C 18:2) & $29.23(1.19)$ & $31.65(0.82)$ & $28.96(1.01)$ & $28.03(1.07)^{*}$ \\
\hline$\alpha$-Linoleic acid (C18:3) & $0.62(0.11)$ & $0.40(0.05)$ & $0.64(0.09)^{*}$ & $0.29(0.03) \dagger$ \\
\hline Arachidonic acid (C20:4) & $6.48(0.42)$ & $6.05(0.60)$ & $5.98(0.62)$ & $5.52(0.27)$ \\
\hline Eicosapentaenoic acid (C20:5) & $1.25(0.10)$ & $1.16(0.20)$ & $1.52(0.35)$ & $4.63(0.44)^{*} \dagger$ \\
\hline Docosapentaenoic acid (C22:5) & $0.29(0.02)$ & $0.26(0.02)$ & $0.24(0.02)$ & $0.42(0.05)^{*} \dagger$ \\
\hline Docosahexaenoic acid (C22:6) & $2.12(0.25)$ & $2.09(0.34)$ & $2.09(0.33)$ & $3.36(0.28)^{*} \dagger$ \\
\hline Remaining fatty acids & $7.99(0.42)$ & $7.86(0.31)$ & $8.06(0.23)$ & $7.61(0.20)$ \\
\hline
\end{tabular}

Values are mean (SEM)

${ }^{*} p \leqslant 0.05$ compared with hypertriglyceridaemic patients at baseline; $\uparrow p \leqslant 0.05$ versus bezafibrate. 
increase in biliary cholesterol saturation, probably due to the simultaneously observed increase in biliary phospholipid concentrations. This observed tendency to increased molar percentage of biliary phospholipids as well as to the increased molar ratio of phospholipids to bile acids on bezafibrate may have been caused by fibrate induced MDR3 gene expression, encoding canalicular phospholipid translocators and biliary phospholipid secretion, as shown in mice. ${ }^{33}$ The increase in the relative amount of phospholipids versus bile salts observed on bezafibrate could be of clinical importance as this ratio has been shown to mediate crystallisation and gall stone formation. $^{34}$

Fish oil non-significantly decreased biliary cholesterol saturation, corroborating an earlier study by Berr and colleagues. ${ }^{17}$ This observation may be linked to fish oil induced changes in the fatty acid distribution of biliary phospholipids: the $\omega-3$ fatty acid fractions increased and partly replaced the linoleic acid fraction. Previously, Berr et al suggested that different biliary phosphatidylcholine species have different cholesterol-phospholipid packaging capacities and demonstrated an inverse correlation between CSI and the eicosapentaenoic acid content of biliary phospholipids. ${ }^{35}$ The fact that we observed only a non-significant decrease in biliary cholesterol saturation might be explained by the fact that the eicosapentaenoic acid fraction partly replaced the phosphatidylcholine-linoleic fraction, which is also associated with low cholesterol saturation. ${ }^{35}$ In addition, differences in the study population could have contributed to the discrepancies in outcome: Berr et al studied gall stone patients with a high average CSI $(1.7)^{17}$ whereas we studied HTG patients without sludge or gall stones with an average CSI of 1.1.

In addition to bile supersaturated with cholesterol, gall bladder dysmotility also contributes to gall stone formation. ${ }^{6}$ We found impaired gall bladder motility, both during exogenous CCK administration and in response to a meal, in HTG patients, which improved on treatment with both bezafibrate and fish oil. Fasting gall bladder motor activity is controlled by hormonal and neural pathways ${ }^{36} 37$ whereas postprandial gall bladder motility is mainly regulated by CCK. $^{38}$ Fasting plasma CCK levels were similar whereas postprandial CCK secretion was higher in HTG patients than in controls, demonstrating that reduced CCK release is not the cause of impaired gall bladder motility in HTG. In contrast, both the results from exogenous CCK induced gall bladder emptying as well as those from regression analysis, characterising the relationship between postprandial gall bladder emptying and integrated CCK levels, point to a decreased sensitivity of the gall bladder to CCK as a cause of impaired gall bladder motility in HTG. The involvement of serum TG levels in this process is further supported by the observed relationship between the magnitude of TG lowering and the increase in gall bladder sensitivity to CCK observed on TG lowering therapy: a larger TG lowering effect concurrent with higher gall bladder sensitivity to CCK was observed on bezafibrate compared with fish oil. The underlying mechanism of this phenomenon is as yet unclear but may encompass HTG induced lipid perturbation of the sarcolemmal lipid bilayer, altering the interaction of CCK with its receptor and/or the CCK receptor-G protein interaction. Another explanation for decreased gall bladder sensitivity to CCK in HTG could be related to cholesterol deposition in the gall bladder. Gall bladder emptying capacity is impaired in subjects with supersaturated bile because of cholesterol deposition in the gall bladder muscularis propria. ${ }^{39}$ However, this explanation is less likely as HTG patients at baseline showed impaired gall bladder motility in comparison with controls, without significant differences in biliary cholesterol saturation. In addition, bezafibrate increased biliary molar percentage cholesterol while gall bladder motility improved compared with baseline.

The observation that TG lowering therapy improves gall bladder motility appears to be restricted to HTG patients: both treatment with bezafibrate in diabetics ${ }^{39}$ and treatment with fish oil in gall stone patients ${ }^{17}$ did not affect gall bladder motility in spite of significant TG lowering effects. Baseline TG levels in diabetics and gall stone patients were however within normal limits, ${ }^{17}{ }^{40}$ far below the average serum TG concentrations of our HTG patients. In addition, the current study showed no difference in gall bladder motility between HTG patients on bezafibrate therapy and controls whereas serum TG levels were still significantly higher in HTG patients than in controls. Apparently, only marked HTG induces gall bladder dysmotility and TG lowering therapy only seems to improve gall bladder dysmotility after reversal of prominently high serum TG levels.

The fact that bezafibrate improves gall bladder motility in HTG patients is of potential clinical relevance. Earlier studies showed an increased incidence of gall stones on fibrate therapy. ${ }^{12-14}$ However, in those studies, the majority of patients were hypercholesterolaemic and not hypertriglyceridaemic. The current study demonstrates that bezafibrate improves gall bladder motility and, in spite of the increase in molar percentage cholesterol, does not cause bile supersaturated with cholesterol in HTG.

The present data indicate that fish oil at a dose of $5 \mathrm{~g} /$ day may be a therapeutic alternative to bezafibrate in HTG patients. Fish oil exerted similar TG lowering capacities and improved gall bladder motility, whereas in contrast with bezafibrate it did not adversely affect biliary cholesterol content. However, further larger long term prospective studies on clinical end points (cardiovascular events, gall stones, pancreatitis) are required to establish the use of fish oil as a therapeutic alternative to fibrates in HTG patients.

\section{ACKNOWLEDGEMENTS}

We thank "Stichting Gastrostart" for their financial support.

\section{Authors' affiliations}

I J A M Jonkers, A H M Smelt, M E Hollum, A E Meinders, Department of General Internal Medicine, Leiden University Medical Centre, PO Box 9600, 2300 RC Leiden, the Netherlands

M Ledeboer, I Biemond, C H B W Lamers, A A M Masclee, Department of Gastroenterology, Leiden University Medical Centre PO Box 9600, 2300 RC Leiden, the Netherlands

F Kuipers, F Stellaard, R Boverhof, Centre for Liver, Intestinal, and Metabolic Diseases, Academic Hospital Groningen, Groningen, the Netherlands

\section{REFERENCES}

1 Hokanson JE, Austin MA. Plasma triglyceride level is a risk factor for cardiovascular disease independent of high-density lipoprotein cholesterol level: a meta-analysis of population-based prospective studies. J Cardiovasc Risk 1996:3:213-19.

2 Toskes PP. Hyperlipidemic pancreatitis. Gastroenterol Clin North Am 1990;19:783-91.

3 Einarsson K, Hellstrom K, Kallner M. Gallbladder disease in hyperlipoproteinaemia. Lancet 1975;1: 484-7.

4 Ahlberg J, Angelin B, Einarsson K, et al. Prevalence of gallbladder disease in hyperlipoproteinemia. Dig Dis Sci 1979;24:459-64.

5 Carey MC, Small DM. The physical chemistry of cholesterol solubility in bile. Relationship to gallstone formation and dissolution in man. J Clin Invest 1978;61:998-1026.

6 Everson GT. Gallbladder function in gallstone disease. Gastroenterol Clin North Am 1991;20:85-110.

7 Duane WC. Abnormal bile acid absorption in familial hypertriglyceridemia. J Lipid Res 1995;36:96-107.

8 Ahlberg J, Angelin B, Einarsson K, et al. Biliary lipid composition in normo- and hyperlipoproteinemia. Gastroenterology 1980;79:90-4

9 Ahmed HA, Jazrawi RP, Goggin PM, et al. Intrahepatic biliary cholesterol and phospholipid transport in humans: effect of obesity and cholesterol cholelithiasis. J Lipid Res 1995;36:2562-73.

10 Grundy SM. Hypertriglyceridemia, atherogenic dyslipidemia, and the metabolic syndrome. Am J Cardiol 1998:81:18-25B.

11 Fruchart JC, Brewer HBJ, Leitersdorf E. Consensus for the use of fibrates in the treatment of dyslipoproteinemia and coronary heart disease. Fibrate Consensus Group. Am J Cardiol 1998;81:912-17.

12 Cooper J, Geizerova H, Oliver MF. Clofibrate and gallstones. Lancet 1975; 1:1083.

13 Bateson MC, Maclean D, Ross PE, et al. Clofibrate therapy and gallstone induction. Am J Dig Dis 1978;23:623-8. 
14 Clofibrate and niacin in coronary heart disease. JAMA 1975 . 231:360-81.

15 Palmer RH. Effects of fibric acid derivatives on biliary lipid composition. Am J Med 1987;83:37-43.

16 Nestel PJ. Fish oil and cardiovascular disease: lipids and arterial function. Am J Clin Nutr 2000;71(suppl):31S.

17 Berr F, Holl J, Jungst D, et al. Dietary N-3 polyunsaturated fatty acids decrease biliary cholesterol saturation in gallstone disease. Hepatology 1992; 16:960-7.

18 Summary of the second report of the National Cholesterol Education Program (NCEP) Expert Panel on Detection, Evaluation, and Treatment of High Blood Cholesterol in Adults (Adult Treatment Panel II). JAMA 1993; 269:3015-23

19 de Man FH, Weverling-Rijnsburger AW, van der Laarse A, et al. Not acute but chronic hypertriglyceridemia is associated with impaired endothelium-dependent vasodilation: reversal after lipid-lowering therapy by atorvastatin. Arterioscler Thromb Vasc Biol 2000;20:744-50

20 Hopman WP, Rosenbusch G, Jansen JB, et al. Gallbladder contraction: effects of fatty meals and cholecystokinin. Radiology 1985;157:37-9.

21 Bligh EG, Dyer WJ. A rapid method of total lipid extraction and purification. Can J Biochem Biophys 1959;37:91 1-17.

22 Gamble W, Vaughan M, Kruth HS, et al. Procedure for determination of free and total cholesterol in micro- or nanogram amounts suitable for studies with cultured cells. J Lipid Res 1978;19:1068-70.

23 Bötcher CFJ, van Gent CM, Pries C. A rapid and sensitive sub micro-phosphorous determination. Anal Chim Acta 1961;24:203-4.

24 Murphy GM, Billing BH, Baron DN. A fluorimetric and enzymatic method for the estimation of serum total bile acids. J Clin Pathol 1970;23:594-8

25 Bandsma RH, Kuipers F, Vonk RJ, et al. The contribution of newly synthesized cholesterol to bile salt synthesis in rats quantified by mass isotopomer distribution analysis. Biochim Biophys Acta $2000 ; 31 ; 1483: 343-51$.

26 Carey MC. Critical tables for calculating the cholesterol saturation of native bile. J Lipid Res 1978;19:945-55.

27 Lepage G, Roy CC. Direct transesterification of all classes of lipids in a one-step reaction. J Lipid Res 1986;27:1 14-20.
28 Muskiet FA, van Doormaal JJ, Martini IA, et al. Capillary gas chromatographic profiling of total long-chain fatty acids and cholesterol in biological materials. J Chromatogr 1983;278:231-44

29 Jansen JB, Lamers CB. Molecular forms of cholecystokinin in plasma from normal and gastrectomized human subjects following a fat meal. Peptides 1987:8:801-5.

30 Jansen JB, Lamers CB. Radioimmunoassay of cholecystokinin in human tissue and plasma. Clin Chim Acta 1983;131:305-16.

31 von Bergmann K, Leiss O. Effect of short-term treatment with bezafibrate and fenofibrate on biliary lipid metabolism in patients with hyperlipoproteinaemia. Eur J Clin Invest 1984;14:150-4.

32 Stahlberg D, Reihner E, Rudling $M$, et al. Influence of bezafibrate on hepatic cholesterol metabolism in gallstone patients: reduced activity of cholesterol 7 alpha-hydroxylase. Hepatology 1995;21:1025-30.

33 Chianale J, Vollrath V, Wielandt AM, et al. Fibrates induce mdr2 gene expression and biliary phospholipid secretion in the mouse. Biochem $J$ 1996;314:781-6.

34 Moschetta A, van Berge Henegouwen GP, Portincasa P, et al. Cholesterol crystalization in model biles. Effects of bile salt and phospholipid species composition. J Lipid Res 2001;42:1273-81.

35 Berr F, Schreiber E, Frick U. Interrelationships of bile acid and phospholipid fatty acid species with cholesterol saturation of duodenal bile in health and gallstone disease. Hepatology 1992;16:71-81.

36 Jebbink MC, Masclee AA, van der Kleij FG, et al. Effect of loxiglumide and atropine on erythromycin-induced reduction in gallbladder volume in human subjects. Hepatology 1992;16:937-42.

37 Fisher RS, Rock E, Malmud LS. Cholinergic effects on gallbladder emptying in humans. Gastroenterology 1985; 89:716-22.

38 Froehlich F, Gonvers JJ, Fried M. Role of nutrient fat and cholecystokinin in regulation of gallbladder emptying in man. Dig Dis Sci 1995:40:529-33.

39 Behar J, Lee KY, Thompson WR, et al. Gallbladder contraction in patients with pigment and cholesterol stones. Gastroenterology 1989;97:1479-84

40 Wilson IR, Hurrell MA, Pattinson NR, et al. The effect of simvastatin and bezafibrate on bile composition and gall-bladder emptying in female non-insulin-dependent diabetics. J Gastroenterol Hepatol 1994;9:447-51. 